\title{
Dynamics of a stochastic delay competition model with imprecise parameters
}

Xin $\mathrm{He}^{\mathrm{a}}$, Meng Liua,b,*

a School of Mathematical Science, Huaiyin Normal University, Huaian 223300, P. R. China.

${ }^{b}$ School of Mathematics and Statistics, Northeast Normal University, Jilin 130024, P. R. China.

Communicated by A. Atangana

\begin{abstract}
This paper is concerned with a two-species delay stochastic competition model with imprecise parameters. We first obtain the thresholds between persistence and extinction for each species. Then we establish sharp sufficient criteria for the existence of a unique ergodic stationary distribution of the model. The effects of imprecise parameters on the persistence, extinction and existence of the stationary distribution are revealed. Finally, we work out some numerical simulations to illustrate the theoretical results. (C)2017 All rights reserved.
\end{abstract}

Keywords: Competition system, stochastic perturbations, imprecise parameters, time delay.

2010 MSC: 92D25, 60H10, 60H30.

\section{Introduction}

Competition is a common phenomenon in the natural world. On the other hand, time delay and stochastic perturbations should not be neglected (see, e.g., $[3,6,19])$. Therefore, it is interesting and important to study stochastic competition models with delay. A classical delay stochastic competition model can be expressed as follows:

$$
\left\{\begin{array}{l}
d y_{1}(t)=y_{1}(t)\left[r_{1}-c_{11} y_{1}(t)-c_{12} y_{2}\left(t-\tau_{1}\right)\right] d t+\sigma_{1} y_{1}(t) d B_{1}(t) \\
d y_{2}(t)=y_{2}(t)\left[r_{2}-c_{21} y_{1}\left(t-\tau_{2}\right)-c_{22} y_{2}(t)\right] d t+\sigma_{2} y_{2}(t) d B_{2}(t)
\end{array}\right.
$$

with initial value:

$$
y(\theta)=\left(y_{1}(\theta), y_{2}(\theta)\right)^{\top}=\left(\eta_{1}(\theta), \eta_{2}(\theta)\right)^{\top} \in \Gamma,
$$

where for $i, j=1,2, j \neq i$, parameters are shown in Table 1 .

\footnotetext{
*Corresponding author

Email address: liumeng0557@163.com (Meng Liu)
}

doi:10.22436/jnsa.010.09.20 
Table 1: Parameters

\begin{tabular}{|l|l|}
\hline$y_{i}(t)$ & Population size of the $i$ th species \\
\hline$r_{i}>0$ & Intrinsic growth rate of the ith species \\
\hline$c_{i i}>0$ & Intra-specific competition rate of the $i$ th species \\
\hline$c_{i j}>0$ & Inter-specific competition rate between the species $i$ and $j$ \\
\hline$\sigma_{i}^{2}$ & Intensity of the white noise \\
\hline$\tau_{i} \geqslant 0$ & Time delay \\
\hline$\left(B_{1}(t), B_{2}(t)\right)^{\top}$ & $\begin{array}{l}\text { A standard Brownian motion defined on a complete probability space } \\
\left(\Omega, \mathcal{F},\left\{\mathcal{F}_{t}\right\}_{t} \geqslant 0, \mathbb{P}\right) \text { with a filtration }\left\{\mathcal{F}_{t}\right\}_{t} \geqslant 0 .\end{array}$ \\
\hline$\Gamma$ & $\begin{array}{l}\text { The family of all bounded and continuous functions from }[-\tau, 0] \text { to } \\
\mathbb{R}_{+}^{2}=\left\{y=\left(y_{1}, y_{2}\right)^{\top} \in \mathbb{R}^{2} \mid y_{i}>0, i=1,2\right\}, \tau=\max \left\{\tau_{1}, \tau_{2}\right\} .\end{array}$ \\
\hline
\end{tabular}

In recent years, model (1.1) and its various generalized forms have been studied extensively, for example, persistence and extinction of model (1.1) were considered in [16]; stability in distribution of model (1.1) was exploited in [13, 15]; optimal control of model (1.1) with harvesting was investigated in [10, 12]; model (1.1) with Lévy jumps was analyzed in [14].

Model (1.1) supposes that all the parameters in the model are precisely known. However, in reality the values of all parameters can not always be known precisely due to the lack of data and mistakes in the measurement process $([21,25])$. Some authors ([20-26]) have claimed that models with imprecise parameters are more realistic. Therefore it is important to consider stochastic delay competition models with imprecise parameters and to reveal the impact of imprecise parameters on the dynamics of the models. However, to the best of our knowledge, no results of this aspect have been reported.

Motivated by these, in this paper we consider the following delay stochastic competition model with interval coefficients:

$$
\left\{\begin{array}{l}
d y_{1}(t)=y_{1}(t)\left[\hat{r}_{1}-\hat{c}_{11} y_{1}(t)-\hat{c}_{12} y_{2}\left(t-\hat{\tau}_{1}\right)\right] d t+\sum_{i=1}^{n} \hat{\sigma}_{1 i} y_{1}(t) d B_{i}(t), \\
d y_{2}(t)=y_{2}(t)\left[\hat{r}_{2}-\hat{c}_{21} y_{1}\left(t-\hat{\tau}_{2}\right)-\hat{c}_{22} y_{2}(t)\right] d t+\sum_{i=1}^{n} \hat{\sigma}_{2 i} y_{2}(t) d B_{i}(t),
\end{array}\right.
$$

with initial condition (1.2). Here, $\left(B_{1}(t), \ldots, B_{n}(t)\right)^{\top}$ is an $n$-dimensional Brownian motion defined on the probability space $\left(\Omega, \mathcal{F},\left\{\mathcal{F}_{t}\right\}_{t \geqslant 0}, \mathbb{P}\right)$; â means the interval counterpart of a, i.e., $\hat{a}=\left[a_{l}, a_{\mathfrak{u}}\right]=\left\{x \in \mathbb{R} \mid a_{l} \leqslant\right.$ $\left.x \leqslant a_{u}\right\}$. For biological reasons, in this paper we suppose that $r_{i l}>0, c_{i j l}>0, \tau_{i l} \geqslant 0, i, j=1,2$. For any $x \in\left[a_{l}, a_{\mathfrak{u}}\right]$, there is a $q \in[0,1]$ such that $x=a_{l}^{1-q} a_{\mathfrak{u}}^{q}$. Hence we shall consider the following model:

$$
\left\{\begin{aligned}
\mathrm{d} y_{1}(t ; q)= & y_{1}(t ; q)\left[r_{1 l}^{1-q} r_{1 u}^{q}-c_{11 l}^{1-q} c_{11 u}^{q} y_{1}(t ; q)-c_{12 l}^{1-q} c_{12 u}^{q} y_{2}\left(t-\tau_{1 l}^{1-q} \tau_{1 u}^{q} ; q\right)\right] d t \\
& +\sum_{i=1}^{n} \sigma_{1 i l}^{1-q} \sigma_{1 i u}^{q} y_{1}(t ; q) d B_{i}(t), \\
d y_{2}(t ; q)= & y_{2}(t ; q)\left[r_{1 l}^{1-q} r_{1 u}^{q}-c_{21 l}^{1-q} c_{21 u}^{q} y_{1}\left(t-\tau_{2 l}^{1-q} \tau_{2 u}^{q} ; q\right)-c_{22 l}^{1-q} c_{22 u}^{q} y_{2}(t ; q)\right] d t \\
& +\sum_{i=1}^{n} \sigma_{2 i l}^{1-q} \sigma_{2 i u}^{q} y_{2}(t ; q) d B_{i}(t),
\end{aligned}\right.
$$

with initial condition (1.2). Clearly, model (1.3) contains the cases that the random noises are correlated or independent.

Remark 1.1. In this paper, we consider the Itô integral instead of Stratonovich integral because model (1.3) is an approximation to age-structured populations ([3]). 
The rest of this paper is organized as follows. In Section 2 we establish the critical value between persistence and extinction for each species. In Section 3 we obtain sharp sufficient criteria for the existence of a unique ergodic stationary distribution of the model. In Section 4 we discuss the effects of imprecise parameters on the persistence, extinction and existence of the stationary distribution of the model with the help of several numerical simulations, and give some concluding remarks.

\section{Persistence and extinction}

For the sake of convenience, we define some notations:

$$
\begin{aligned}
\langle f(t)\rangle & =t^{-1} \int_{0}^{t} f(s) d s, b_{i}(q)=r_{i l}^{1-q} r_{i u}^{q}-\sum_{k=1}^{n} \sigma_{i k l}^{2(1-q)} \sigma_{i k u}^{2 q} / 2, i=1,2, \\
\Delta(q) & =c_{111}^{1-q} c_{11 u}^{q} c_{22 l}^{1-q} c_{22 u}^{q}-c_{12 l}^{1-q} c_{12 u}^{q} c_{21 l}^{1-q} c_{21 u^{\prime}}^{q} \\
\Delta_{1}(q) & =b_{1}(q) c_{22 l}^{1-q} c_{22 u}^{q}-b_{2}(q) c_{12 l}^{1-q} c_{12 u^{\prime}}^{q} \Delta_{2}(q)=b_{2}(q) c_{11 l}^{1-q} c_{11 u}^{q}-b_{1}(q) c_{21 l}^{1-q} c_{21 u}^{q} .
\end{aligned}
$$

To begin with, let us prepare several lemmas.

Lemma 2.1 ([17]). Let $\rho(\mathrm{t}) \in \mathrm{C}\left(\Omega \times[0,+\infty), \mathbb{R}_{+}\right)$.

(i) If there are two positive constants $\mathrm{T}$ and $\beta_{0}$ such that for all $\mathrm{t} \geqslant \mathrm{T}$,

$$
\ln \rho(t) \leqslant \beta t-\beta_{0} \int_{0}^{t} \rho(s) d s+\sum_{i=1}^{n} \alpha_{i} B_{i}(t),
$$

where $\alpha_{i}, i=1, \ldots, n$ are constants, then

$$
\left\{\begin{array}{l}
\limsup _{t \rightarrow+\infty}\langle\rho(t)\rangle \leqslant \beta / \beta_{0}, \quad \text { a.s. (almost surely), if } \beta \geqslant 0 ; \\
\lim _{t \rightarrow+\infty} \rho(t)=0, \quad \text { a.s., } \quad \text { if } \beta<0,
\end{array}\right.
$$

(ii) If there are three positive constants $\mathrm{T}, \beta$, and $\beta_{0}$ such that for all $\mathrm{t} \geqslant \mathrm{T}$,

$$
\ln \rho(t) \geqslant \beta t-\beta_{0} \int_{0}^{t} \rho(s) d s+\sum_{i=1}^{n} \alpha_{i} B_{i}(t),
$$

then

$$
\liminf _{t \rightarrow+\infty}\langle\rho(t)\rangle \geqslant \beta / \beta_{0}, \quad \text { a.s.. }
$$

Lemma 2.2. For any given initial value $\eta(\theta) \in \Gamma$, model (1.3) has a unique global positive solution $\mathrm{y}(\mathrm{t} ; \mathrm{q})=$ $\left(y_{1}(t ; q), y_{2}(t ; q)\right)$ on $t \geqslant 0$ a.s., and

$$
\limsup _{t \rightarrow+\infty} \frac{\ln y_{i}(t ; q)}{\ln t} \leqslant 1, \text { a.s., } i=1,2
$$

In addition, for all $\mathrm{p}>0$, there is a positive constant $\mathrm{K}(\mathrm{p})$ such that

$$
\limsup _{t \rightarrow \infty} \mathbb{E}\left[y_{i}^{p}(t ; q)\right] \leqslant K(p), i=1,2 .
$$

Proof. Clearly, the coefficients of model (1.3) are locally Lipschitz continuous; hence for any given initial condition $\eta(\theta) \in \Gamma$, model (1.3) has a unique local solution $y(t ; q)$ for $t \in\left[0, \tau_{e}\right)$, where $\tau_{e}$ represents the explosion time. For any $t \in\left[0, \tau_{e}\right)$, 


$$
\begin{aligned}
y_{1}(t ; q)= & y_{1}(0) \exp \left\{\int_{0}^{t}\left(b_{1}(q)-c_{11 l}^{1-q} c_{11 u}^{q} y_{1}(s ; q)-c_{12 l}^{1-q} c_{12 u}^{q} y_{2}\left(s-\tau_{1 l}^{1-q} \tau_{1 u}^{q} ; q\right)\right) d s\right. \\
& \left.+\sum_{i=1}^{n} \int_{0}^{t} \sigma_{1 i l}^{1-q} \sigma_{1 i u}^{q} y_{1}(s ; q) d B_{i}(s)\right\}, \\
y_{2}(t ; q)= & y_{2}(0) \exp \left\{\int_{0}^{t}\left(b_{2}(q)-c_{21 l}^{1-q} c_{21 u}^{q} y_{1}\left(s-\tau_{2 l}^{1-q} \tau_{2 u}^{q} ; q\right)-c_{22 l}^{1-q} c_{22 u}^{q} y_{2}(s ; q)\right) d s\right. \\
& \left.+\sum_{i=1}^{n} \int_{0}^{t} \sigma_{2 i l}^{1-q} \sigma_{2 i u}^{q} y_{2}(s ; q) d B_{i}(s)\right\} .
\end{aligned}
$$

Therefore, $y_{i}(t ; q)>0$ for $t \in\left[0, \tau_{e}\right), i=1,2$. Now let us prove $\tau_{e}=+\infty$. To this end, we consider the following auxiliary equations:

$$
\begin{aligned}
\mathrm{d} x_{i}(t ; q)= & x_{i}(t ; q)\left[r_{i l}^{1-q} r_{i u}^{q}-c_{i i l}^{1-q} c_{i i u}^{q} x_{i}(t ; q)\right] d t+\sum_{k=1}^{n} \sigma_{i k l}^{1-q} \sigma_{i k u}^{q} x_{i}(t ; q) d B_{k}(t), i=1,2, \\
d z_{j}(t ; q)= & z_{j}(t ; q)\left[r_{j l}^{1-q} r_{j u}^{q}-c_{j j l}^{1-q} c_{j j u}^{q} z_{j}(t ; q)-c_{j i l}^{1-q} c_{j i u}^{q} x_{i}\left(t-\tau_{j l}^{1-q} \tau_{j u}^{q} ; q\right)\right] d t \\
& +\sum_{k=1}^{n} \sigma_{j k l}^{1-q} \sigma_{j k u}^{q} z_{j}(t ; q) d B_{k}(t), j=1,2, j \neq i,
\end{aligned}
$$

with initial data $x(\theta)=z(\theta)=y(\theta)$. According to the comparison theorem [7], we have

$$
z_{i}(t ; q) \leqslant y_{i}(t ; q) \leqslant x_{i}(t ; q), t \in\left[0, \tau_{e}\right), i=1,2 .
$$

On the other hand, according to [8], the explicit solutions of equations (2.3) and (2.4) can be expressed as follows:

$$
\begin{aligned}
& x_{i}(t ; q)=\frac{\exp \left\{b_{i}(p) t+\sum_{k=1}^{n} \sigma_{i k l}^{1-q} \sigma_{i k u}^{q} B_{k}(t)\right\}}{x_{i}^{-1}(0)+c_{i i l}^{1-q} c_{i i u}^{q} \int_{0}^{t} \exp \left\{b_{i}(p) s+\sum_{k=1}^{n} \sigma_{i k l}^{1-q} \sigma_{i k u}^{q} B_{k}(s)\right\} d s}, i=1,2, \\
& z_{i}(t ; q)=\frac{\exp \left\{b_{i}(p) t-c_{i j l}^{1-q} c_{i j u}^{q} \int_{0}^{t} x_{j}\left(s-\tau_{i l}^{1-q} \tau_{i u}^{q} ; q\right) d s+\sum_{k=1}^{n} \sigma_{i k l}^{1-q} \sigma_{i k u}^{q} B_{k}(t)\right\}}{z_{i}^{-1}(0)+c_{i i l}^{1-q} c_{i i u}^{q} \int_{0}^{t} \exp \left\{b_{i}(p) s-c_{i j l}^{1-q} c_{i j u}^{q} \int_{0}^{s} x_{j}\left(\mu-\tau_{i l}^{1-q} \tau_{i u}^{q} ; q\right) d \mu+\sum_{k=1}^{n} \sigma_{i k l}^{1-q} \sigma_{i k u}^{q} B_{k}(s)\right\} d s}, j \neq i .
\end{aligned}
$$

Note that $x_{\mathfrak{i}}(t ; q)>0$ and $z_{\mathfrak{i}}(t ; q)>0$ exist on $[0,+\infty)$, hence $\tau_{e}=+\infty$.

Now let us show (2.1) and (2.2). In fact, according to the results in [9],

$$
\limsup _{t \rightarrow+\infty} \frac{\ln x_{i}(t ; q)}{\ln t} \leqslant 1, \text { a.s., } i=1,2,
$$

and for all $p>0$, there is a positive constant $K(p)$ such that

$$
\limsup _{t \rightarrow \infty} \mathbb{E}\left[x_{i}^{p}(t ; q)\right] \leqslant K(p), i=1,2 .
$$

Hence the desired assertions (2.1) and (2.2) follow from (2.5).

The aim of this section is to study the persistence and extinction of model (1.3). If $b_{i}(q)<0$, then the species $i$ in model (1.3) goes to extinction, i.e., $\lim _{t \rightarrow+\infty} y_{i}(t ; q)=0$, a.s., $i=1,2$ (the proof is standard and hence is omitted (see, e.g. [17]). Hence from now on, we always suppose that $b_{1}(q)>0$ and $b_{2}(q)>0$. Besides, we also suppose the following.

Assumption 2.3. $\Delta(\mathrm{q})>0$, that is to say, the intra-specific competition is stronger than the inter-specific competition. 
Remark 2.4. If $\Delta(q)>0$, it is easy to show that $\Delta_{1}(q)<0$ and $\Delta_{2}(q)<0$ will not hold simultaneously.

Now we are in the position to state and prove our first main result.

Theorem 2.5. Suppose that $\mathrm{b}_{1}(\mathrm{q})>0, \mathrm{~b}_{2}(\mathrm{q})>0$ and Assumption 2.3 holds.

(i) If $\Delta_{1}(q)>0$ and $\Delta_{2}(q)<0$, then $y_{2}$ goes to extinction a.s. and $y_{1}$ is persistent in the mean a.s.:

$$
\lim _{\mathrm{t} \rightarrow+\infty} \mathrm{t}^{-1} \int_{0}^{\mathrm{t}} \mathrm{y}_{1}(\mathrm{~s} ; \mathrm{q}) \mathrm{d} s=\frac{\mathrm{b}_{1}(\mathrm{q})}{\mathrm{c}_{11 \mathrm{l}}^{1-\mathrm{q}} \mathrm{c}_{11 \mathrm{u}}^{\mathrm{q}}}, \text { a.s.; }
$$

(ii) If $\Delta_{1}(\mathrm{q})<0$ and $\Delta_{2}(\mathrm{q})>0$, then $\mathrm{y}_{1}$ goes to extinction a.s. and $\mathrm{y}_{2}$ is persistent in the mean a.s.:

$$
\lim _{\mathrm{t} \rightarrow+\infty} \mathrm{t}^{-1} \int_{0}^{\mathrm{t}} \mathrm{y}_{2}(\mathrm{~s} ; \mathrm{q}) \mathrm{d} s=\frac{\mathrm{b}_{2}(\mathrm{q})}{\mathrm{c}_{22 \mathrm{l}}^{1-\mathrm{q}} \mathrm{c}_{22 \mathrm{u}}^{\mathrm{q}}}, \text { a.s.; }
$$

(iii) If $\Delta_{1}(\mathrm{q})>0$ and $\Delta_{2}(\mathrm{q})>0$, then both $\mathrm{y}_{1}$ and $\mathrm{y}_{2}$ are persistent in the mean a.s.:

$$
\lim _{\mathrm{t} \rightarrow+\infty} \mathrm{t}^{-1} \int_{0}^{\mathrm{t}} \mathrm{y}_{1}(\mathrm{~s} ; \mathrm{q}) \mathrm{d} \mathrm{s}=\frac{\Delta_{1}(\mathrm{q})}{\Delta(\mathrm{q})}, \lim _{\mathrm{t} \rightarrow+\infty} \mathrm{t}^{-1} \int_{0}^{\mathrm{t}} \mathrm{y}_{2}(\mathrm{~s} ; \mathrm{q}) \mathrm{d} \mathrm{s}=\frac{\Delta_{2}(\mathrm{q})}{\Delta(\mathrm{q})}, \text { a.s.. }
$$

Proof. Applying Itô's formula to (2.3) yields

$$
\ln x_{i}(t ; q)-\ln x_{i}(0)=b_{i}(q) t-c_{i i l}^{1-q} c_{i i u}^{q} \int_{0}^{t} x_{i}(s ; q) d s+\sum_{k=1}^{n} \sigma_{i k l}^{1-q} \sigma_{i k u}^{q} B_{k}(t), i=1,2 .
$$

Note that $b_{i}(q)>0$, it then follows from Lemma 2.1 that

$$
\frac{b_{i}(q)}{c_{i i l}^{1-q} c_{i i u}^{q}} \leqslant \liminf _{t \rightarrow+\infty}\left\langle x_{i}(t ; q)\right\rangle \leqslant \limsup _{t \rightarrow+\infty}\left\langle x_{i}(t ; q)\right\rangle \leqslant \frac{b_{i}(q)}{c_{i i l}^{1-q} c_{i i u}^{q}}, \text { a.s., } i=1,2 .
$$

Therefore

$$
\lim _{t \rightarrow+\infty}\left\langle x_{i}(t ; q)\right\rangle=\lim _{t \rightarrow+\infty} t^{-1} \int_{0}^{t} x_{i}(s ; q) d s=\frac{b_{i}(q)}{c_{i i l}^{1-q} c_{i i u}^{q}}, \text { a.s., } i=1,2 .
$$

Consequently,

$$
\begin{aligned}
\lim _{t \rightarrow+\infty} t^{-1} \int_{t-\tau_{j l}^{1-q} \tau_{j u}^{q}}^{t} x_{i}(s ; q) d s & =\lim _{t \rightarrow+\infty} t^{-1}\left[\int_{0}^{t} x_{i}(s ; q) d s-\int_{0}^{t-\tau_{j l}^{1-q} \tau_{j u}^{q}} x_{i}(s ; q) d s\right] \\
& =\frac{b_{i}(q)}{c_{i i l}^{1-q} c_{i i u}^{q}}-\frac{b_{i}(q)}{c_{i i l}^{1-q} c_{i i u}^{q}}=0, \text { a.s., } i, j=1,2, j \neq i .
\end{aligned}
$$

In light of (2.5), we have

$$
\lim _{t \rightarrow+\infty} t^{-1} \int_{t-\tau_{j l}^{1-q} \tau_{j u}^{q}}^{t} y_{i}(s ; q) d s=0, \text { a.s., } i, j=1,2, j \neq i .
$$

Applying Itô's formula to (1.3) yields

$$
\begin{aligned}
& \ln y_{1}(t ; q)-\ln y_{1}(0) \\
& =b_{1}(q) t-c_{11 l}^{1-q} c_{11 u}^{q} \int_{0}^{t} y_{1}(s ; q) d s-c_{12 l}^{1-q} c_{12 u}^{q} \int_{0}^{t} y_{2}\left(s-\tau_{1 l}^{1-q} \tau_{1 u}^{q} ; q\right) d s+\sum_{i=1}^{n} \sigma_{1 i l}^{1-q} \sigma_{1 i u}^{q} B_{i}(t) \\
& =b_{1}(q) t-c_{12 l}^{1-q} c_{12 u}^{q} \int_{0}^{t} y_{2}(s ; q) d s+c_{12 l}^{1-q} c_{12 u}^{q}\left[\int_{t-\tau_{1 l}^{1-q} \tau_{1 u}^{q}}^{t} y_{2}(s ; q) d s-\int_{-\tau_{1 l}^{1-q} \tau_{1 u}^{q}}^{0} y_{2}(s ; q) d s\right] \\
& \quad-c_{11 l}^{1-q} c_{11 u}^{q} \int_{0}^{t} y_{1}(s ; q) d s+\sum_{i=1}^{n} \sigma_{1 i l}^{1-q} \sigma_{1 i u}^{q} B_{i}(t) .
\end{aligned}
$$




$$
\begin{aligned}
& \ln y_{2}(t ; q)-\ln y_{2}(0) \\
& =b_{2}(q) t-c_{21 l}^{1-q} c_{21 u}^{q} \int_{0}^{t} y_{1}\left(s-\tau_{2 l}^{1-q} \tau_{2 u}^{q} ; q\right) d s-c_{22 l}^{1-q} c_{22 u}^{q} \int_{0}^{t} y_{2}(s ; q) d s+\sum_{i=1}^{n} \sigma_{2 i l}^{1-q} \sigma_{2 i u}^{q} B_{i}(t) \\
& =b_{2}(q) t-c_{21 l}^{1-q} c_{21 u}^{q} \int_{0}^{t} y_{1}(s ; q) d s+c_{21 l}^{1-q} c_{21 u}^{q}\left[\int_{t-\tau_{2 l}^{1-q} \tau_{2 u}^{q}}^{t} y_{1}(s ; q) d s-\int_{-\tau_{2 l}^{1-q} \tau_{2 u}^{q}}^{q} y_{1}(s ; q) d s\right] \\
& \quad-c_{22 l}^{1-q} c_{22 u}^{q} \int_{0}^{t} y_{2}(s ; q) d s+\sum_{i=1}^{n} \sigma_{2 i l}^{1-q} \sigma_{2 i u}^{q} B_{i}(t) .
\end{aligned}
$$

Computing $(2.11) \times c_{11 l}^{1-q} c_{11 u}^{q}-(2.10) \times c_{21 l}^{1-q} c_{21 u}^{q}$, we can see that

$$
\begin{aligned}
c_{11 l}^{1-q} c_{11 u}^{q} \ln \frac{y_{2}(t ; q)}{y_{2}(0)}= & c_{11 l}^{1-q} c_{11 u}^{q} c_{21 l}^{1-q} c_{21 u}^{q}\left[\int_{t-\tau_{2 l}^{1-q} \tau_{2 u}^{q}}^{t} y_{1}(s ; q) d s-\int_{-\tau_{2 l}^{1-q} \tau_{2 u}^{q}}^{0} y_{1}(s ; q) d s\right] \\
& -c_{21 l}^{1-q} c_{21 u}^{q} c_{12 l}^{1-q} c_{12 u}^{q}\left[\int_{t-\tau_{1 l}^{1-q} \tau_{1 u}^{q}}^{t} y_{2}(s ; q) d s-\int_{-\tau_{1 l}^{1-q} \tau_{1 u}^{q}} y_{2}(s ; q) d s\right] \\
& +c_{21 l}^{1-q} c_{21 u}^{q} \ln \frac{x_{1}(t ; q)}{x_{1}(0)}+\Delta_{2}(q) t-\Delta(q) \int_{0}^{t} y_{2}(s ; q) d s \\
& -c_{21 l}^{1-q} c_{21 u}^{q} \sum_{i=1}^{n} \sigma_{1 i l}^{1-q} \sigma_{1 i u}^{q} B_{i}(t)+c_{11 l}^{1-q} c_{11 u}^{q} \sum_{i=1}^{n} \sigma_{2 i l}^{1-q} \sigma_{2 i u}^{q} B_{i}(t) .
\end{aligned}
$$

According to (2.1) and (2.9), for arbitrary $\varepsilon>0$, there exists a $T>0$ such that for $t \geqslant T$,

$$
\begin{aligned}
& t^{-1} c_{21 l}^{1-q} c_{21 u}^{q} \ln \frac{y_{1}(t ; q)}{y_{1}(0)} \leqslant \varepsilon / 4, \\
& t^{-1} c_{11 l}^{1-q} c_{11 u}^{q} \ln y_{2}(0) \leqslant \varepsilon / 4 \\
& t^{-1} c_{11 l}^{1-q} c_{11 u}^{q} c_{21 l}^{1-q} c_{21 u}^{q}\left[\int_{t-\tau_{2 l}^{1-q} \tau_{2 u}^{q}}^{t} y_{1}(s ; q) d s-\int_{-\tau_{2 l}^{1-q} \tau_{2 u}^{q}}^{0} y_{1}(s ; q) d s\right] \leqslant \varepsilon / 4, \\
& -t^{-1} c_{21 l}^{1-q} c_{21 u}^{q} c_{12 l}^{1-q} c_{12 u}^{q}\left[\int_{t-\tau_{1 l}^{1-q} \tau_{1 u}^{q}}^{t} y_{2}(s ; q) d s-\int_{-\tau_{1 l}^{1-q} \tau_{1 u}^{q}}^{q} y_{2}(s ; q) d s\right] \leqslant \varepsilon / 4 .
\end{aligned}
$$

When the above inequalities are used in (2.12), we can see that for $t>T$,

$$
\begin{aligned}
c_{11 l}^{1-q} c_{11 u}^{q} \ln y_{2}(t ; q) \leqslant & \left(\Delta_{2}(q)+\varepsilon\right) t-\Delta(q) \int_{0}^{t} y_{2}(s ; q) d s \\
& -c_{21 l}^{1-q} c_{21 u}^{q} \sum_{i=1}^{n} \sigma_{1 i l}^{1-q} \sigma_{1 i u}^{q} B_{i}(t)+c_{11 l}^{1-q} c_{11 u}^{q} \sum_{i=1}^{n} \sigma_{2 i l}^{1-q} \sigma_{2 i u}^{q} B_{i}(t) .
\end{aligned}
$$

Similarly, computing $(2.10) \times c_{22 l}^{1-q} c_{22 u}^{q}-(2.11) \times c_{12 l}^{1-q} c_{12 u}^{q}$ results in

$$
\begin{aligned}
c_{22 l}^{1-q} c_{22 u}^{q} \ln \frac{y_{1}(t ; q)}{y_{1}(0)}= & c_{22 l}^{1-q} c_{22 u}^{q} c_{12 l}^{1-q} c_{12 u}^{q}\left[\int_{t-\tau_{1 l}^{1-q} \tau_{1 u}^{q}}^{t} y_{2}(s ; q) d s-\int_{-\tau_{1 l}^{1-q} \tau_{1 u}^{q}}^{0} y_{2}(s ; q) d s\right] \\
& -c_{12 l}^{1-q} c_{12 u}^{q} c_{21 l}^{1-q} c_{21 u}^{q}\left[\int_{t-\tau_{2 l}^{1-q} \tau_{2 u}^{q}}^{t} y_{1}(s ; q) d s-\int_{-\tau_{2 l}^{1-q} \tau_{2 u}^{q}}^{q^{q}} y_{1}(s ; q) d s\right] \\
& +c_{12 l}^{1-q} c_{12 u}^{q} \ln \frac{y_{2}(t ; q)}{y_{2}(0)}+\Delta_{1}(q) t-\Delta(q) \int_{0}^{t} y_{1}(s ; q) d s \\
& +c_{22 l}^{1-q} c_{22 u}^{q} \sum_{i=1}^{n} \sigma_{1 i l}^{1-q} \sigma_{1 i u}^{q} B_{i}(t)-c_{12 l}^{1-q} c_{12 u}^{q} \sum_{i=1}^{n} \sigma_{2 i l}^{1-q} \sigma_{2 i u}^{q} B_{i}(t) .
\end{aligned}
$$


Similar to (2.13), by virtue of (2.14) we can observe that for $t>T$,

$$
\begin{aligned}
c_{22 l}^{1-q} c_{22 u}^{q} \ln y_{1}(t ; q) \leqslant & \left(\Delta_{1}(q)+\varepsilon\right) t-\Delta(q) \int_{0}^{t} y_{1}(s ; q) d s \\
& +c_{22 l}^{1-q} c_{22 u}^{q} \sum_{i=1}^{n} \sigma_{1 i l}^{1-q} \sigma_{1 i u}^{q} B_{i}(t)-c_{12 l}^{1-q} c_{12 u}^{q} \sum_{i=1}^{n} \sigma_{2 i l}^{1-q} \sigma_{2 i u}^{q} B_{i}(t) .
\end{aligned}
$$

(i). Since $\Delta_{2}(q)<0$, then we can choose $\varepsilon$ sufficiently small such that $\Delta_{2}(q)+\varepsilon<0$. Applying (i) in Lemma 2.1 to (2.13) gives $\lim _{t \rightarrow+\infty} y_{2}(t ; q)=0$, a.s.. The proof of (2.6) is similar to that of (2.8) and hence is omitted.

The proof of (ii) is similar to that of (i) and we also omit it.

Now we are in the position to prove (iii). Note that $\Delta_{2}(q)>0$, according to (2.13) and Lemma 2.1, we obtain

$$
\limsup _{t \rightarrow+\infty}\left\langle y_{2}(t ; q)\right\rangle \leqslant \frac{\Delta_{2}(q)+\varepsilon}{\Delta(q)}, \text { a.s.. }
$$

As an application of the arbitrariness of $\varepsilon$, one can observe that

$$
\limsup _{t \rightarrow+\infty}\left\langle y_{2}(t ; q)\right\rangle \leqslant \frac{\Delta_{2}(q)}{\Delta(q)}, \text { a.s.. }
$$

In the same way, by (2.15), Lemma 2.1, and the arbitrariness of $\varepsilon$, we have

$$
\limsup _{t \rightarrow+\infty}\left\langle y_{1}(t ; q)\right\rangle \leqslant \frac{\Delta_{1}(q)}{\Delta(q)}, \text { a.s.. }
$$

Let $\varepsilon<c_{11 l}^{1-q} c_{11 u}^{q} \frac{\Delta_{1}(q)}{\Delta(q)}$. Substituting (2.9) and (2.16) into (2.10) results in that for sufficiently large $t$,

$$
\begin{aligned}
t^{-1} \ln y_{1}(t ; q)= & t^{-1} \ln y_{1}(0)+b_{1}(q)-c_{11 l}^{1-q} c_{11 u}^{q}\left\langle y_{1}(t ; q)\right\rangle-c_{12 l}^{1-q} c_{12 u}^{q}\left\langle y_{2}(t ; q)\right\rangle+\sum_{i=1}^{n} \sigma_{1 i l}^{1-q} \sigma_{1 i u}^{q} B_{i}(t) / t \\
& +c_{12 l}^{1-q} c_{12 u}^{q} t^{-1}\left[\int_{t-\tau_{1 l}^{1-q} \tau_{1 u}^{q}}^{t} y_{2}(s ; q) d s-\int_{-\tau_{1 l}^{1-q} \tau_{1 u}^{q}}^{0} y_{2}(s ; q) d s\right] \\
\geqslant & b_{1}(q)-\varepsilon-c_{11 l}^{1-q} c_{11 u}^{q}\left\langle y_{1}(t ; q)\right\rangle-c_{12 l}^{1-q} c_{12 u}^{q} \limsup _{t \rightarrow+\infty}\left\langle y_{2}(t ; q)\right\rangle+\sum_{i=1}^{n} \sigma_{1 i l}^{1-q} \sigma_{1 i u}^{q} B_{i}(t) / t \\
\geqslant & b_{1}(q)-\varepsilon-c_{11 l}^{1-q} c_{11 u}^{q}\left\langle y_{1}(t ; q)\right\rangle-c_{12 l}^{1-q} c_{12 u}^{q} \frac{\Delta_{2}(q)}{\Delta(q)}+\sum_{i=1}^{n} \sigma_{1 i l}^{1-q} \sigma_{1 i u}^{q} B_{i}(t) / t \\
= & c_{11 l}^{1-q} c_{11 u}^{q} \frac{\Delta_{1}(q)}{\Delta(q)}-\varepsilon-c_{11 l}^{1-q} c_{11 u}^{q}\left\langle y_{1}(t ; q)\right\rangle+\sum_{i=1}^{n} \sigma_{1 i l}^{1-q} \sigma_{1 i u}^{q} B_{i}(t) / t .
\end{aligned}
$$

By virtue of (ii) in Lemma 2.1 and the arbitrariness of $\varepsilon$, one can observe that

$$
\liminf _{t \rightarrow+\infty}\left\langle y_{1}(t ; q)\right\rangle \geqslant \frac{\Delta_{1}(q)}{\Delta(q)}, \text { a.s.. }
$$

In the same way, substituting (2.9) and (2.17) into (2.11), we obtain

$$
\liminf _{t \rightarrow+\infty}\left\langle y_{2}(t ; q)\right\rangle \geqslant \frac{\Delta_{2}(q)}{\Delta(q)}, \text { a.s.. }
$$

Then the desired assertion (2.7) follows. 


\section{Stationary distribution}

In this section, we will consider the existence of a unique stationary distribution of model (1.3), i.e., to prove that there is a probability measure $v$ with support $\mathbb{R}_{+}^{2}$ such that for any initial data $\eta \in \Gamma$, the transition probability $p(t, \eta, \cdot ; q)$ of $y(t ; q)$ converges weakly to $v$ as $t \rightarrow+\infty$ (see e.g. [18]). To this end, we introduce a technical assumption.

Assumption 3.1. $c_{11 l}^{1-q} c_{11 u}^{q}>c_{21 l}^{1-q} c_{21 u^{\prime}}^{q} c_{22 l}^{1-q} c_{22 u}^{q}>c_{12 l}^{1-q} c_{12 u}^{q}$.

Remark 3.2. Clearly, under Assumption 3.1, we have $\Delta(q)>0$ and Remark 2.4.

Theorem 3.3. Let Assumption 3.1 holds.

(a) If $\Delta_{1}(\mathrm{q})>0$ and $\Delta_{2}(\mathrm{q})>0$, then the distribution of $\mathrm{y}(\mathrm{t} ; \mathrm{q})$ weakly converges to a unique distribution $v$ which is ergodic:

$$
\int_{\mathbb{R}_{+}^{2}} \xi_{i} v\left(d \xi_{1}, d \xi_{2} ; q\right)=\lim _{t \rightarrow+\infty}\left\langle y_{i}(t ; q)\right\rangle=\frac{\Delta_{i}(q)}{\Delta(q)}, i=1,2 .
$$

(b) If $\Delta_{1}(q) \Delta_{2}(q)<0$, then model (1.3) does not have a stationary distribution.

Proof. (a). The proof is divided into three parts.

Part 1. In this part, let us prove

$$
\lim _{t \rightarrow \infty} \mathbb{E}\left|y_{i}(t ; q ; \eta)-y_{i}(t ; q ; \psi)\right|=0, i=1,2,
$$

where $y(t ; q ; \eta)$ and $y(t ; q ; \psi)$ are any two solutions of $(1.3)$ with initial values $\eta(\theta) \in \Gamma$ and $\psi(\theta) \in \Gamma$, respectively. Define

$$
\begin{aligned}
V(t ; q)= & \sum_{i=1}^{2}\left|\ln y_{i}(t ; q ; \eta)-\ln y_{i}(t ; q ; \psi)\right|+c_{12 l}^{1-q} c_{12 u}^{q} \int_{t-\tau_{1 l}^{1-q} \tau_{1 u}^{q}}^{t}\left|y_{2}(s ; q ; \eta)-y_{2}(s ; q ; \psi)\right| d s \\
& +c_{21 l}^{1-q} c_{21 u}^{q} \int_{t-\tau_{2 l}^{1-q} \tau_{2 u}^{q}}^{t}\left|y_{1}(s ; q ; \eta)-y_{1}(s ; q ; \psi)\right| d s .
\end{aligned}
$$

According to Itô's formula, one can see that

$$
\begin{aligned}
& d V(t ; q)=\operatorname{sgn}\left(y_{1}(t ; q ; \eta)-y_{1}(t ; q ; \psi)\right)\left[-c_{11 l}^{1-q} c_{11 u}^{q}\left(y_{1}(t ; q ; \eta)-y_{1}(t ; q ; \psi)\right)\right. \\
& \left.-c_{12 l}^{1-q} c_{12 u}^{q}\left(y_{2}\left(t-\tau_{1 l}^{1-q} \tau_{1 u}^{q} ; q ; \eta\right)-y_{2}\left(t-\tau_{1 l}^{1-q} \tau_{1 u}^{q} ; q ; \psi\right)\right)\right] d t \\
& +\operatorname{sgn}\left(y_{2}(t ; q ; \eta)-y_{2}(t ; q ; \psi)\right)\left[-c_{21 l}^{1-q} c_{21 u}^{q}\left(y_{1}\left(t-\tau_{2 l}^{1-q} \tau_{2 u}^{q} ; q ; \eta\right)\right.\right. \\
& \left.\left.-y_{1}\left(t-\tau_{2 l}^{1-q} \tau_{2 u}^{q} ; q ; \psi\right)\right)-c_{22 l}^{1-q} c_{22 u}^{q}\left(y_{2}(t ; q ; \eta)-y_{2}(t ; q ; \psi)\right)\right] d t \\
& +\sum_{i=1}^{2} \sum_{j=1, j \neq i}^{2} c_{i j l}^{1-q} c_{i j u}^{q}\left|y_{j}(t ; q ; \eta)-y_{j}(t ; q ; \psi)\right| d t \\
& -\sum_{i=1}^{2} \sum_{j=1, j \neq i}^{2} c_{i j l}^{1-q} c_{i j u}^{q}\left|y_{j}\left(t-\tau_{i l}^{1-q} \tau_{i u}^{q} ; q ; \eta\right)-y_{j}\left(t-\tau_{i l}^{1-q} \tau_{i u}^{q} ; q ; \psi\right)\right| d t \\
& \leqslant-\sum_{i=1}^{2} c_{i i l}^{1-q} c_{i i u}^{q}\left|y_{i}(t ; q ; \eta)-y_{i}(t ; q ; \psi)\right| d t
\end{aligned}
$$




$$
\begin{aligned}
& +\sum_{i=1}^{2} \sum_{j=1, j \neq i}^{2} c_{i j l}^{1-q} c_{i j u}^{q}\left|y_{j}\left(t-\tau_{i l}^{1-q} \tau_{i u}^{q} ; q ; \eta\right)-y_{j}\left(t-\tau_{i l}^{1-q} \tau_{i u}^{q} ; q ; \psi\right)\right| d t \\
& +\sum_{i=1}^{2} \sum_{j=1, j \neq i}^{2} c_{i j l}^{1-q} c_{i j u}^{q}\left|y_{j}(t ; q ; \eta)-y_{j}(t ; q ; \psi)\right| d t \\
& -\sum_{i=1}^{2} \sum_{j=1, j \neq i}^{2} c_{i j l}^{1-q} c_{i j u}^{q}\left|y_{j}\left(t-\tau_{i l}^{1-q} \tau_{i u}^{q} ; q ; \eta\right)-y_{j}\left(t-\tau_{i l}^{1-q} \tau_{i u}^{q} ; q ; \psi\right)\right| d t \\
& =-\left[c_{11 l}^{1-q} c_{11 u}^{q}-c_{21 l}^{1-q} c_{21 u}^{q}\right]\left|y_{1}(t ; q ; \eta)-y_{1}(t ; q ; \psi)\right| d t \\
& -\left[c_{22 l}^{1-q} c_{22 u}^{q}-c_{12 l}^{1-q} c_{12 u}^{q}\right]\left|y_{2}(t ; q ; \eta)-y_{2}(t ; q ; \psi)\right| d t .
\end{aligned}
$$

Hence

$$
\begin{aligned}
\mathbb{E}(V(t ; q)) \leqslant & V(0)-\left[c_{11 l}^{1-q} c_{11 u}^{q}-c_{21 l}^{1-q} c_{21 u}^{q}\right] \int_{0}^{t} \mathbb{E}\left|y_{1}(s ; q ; \eta)-y_{1}(s ; q ; \psi)\right| d s \\
& -\left[c_{22 l}^{1-q} c_{22 u}^{q}-c_{12 l}^{1-q} c_{12 u}^{q}\right] \int_{0}^{t} \mathbb{E}\left|y_{2}(s ; q ; \eta)-y_{2}(s ; q ; \psi)\right| d s .
\end{aligned}
$$

It then follows from $V(t ; q) \geqslant 0$ that

$$
\begin{aligned}
& {\left[c_{11 l}^{1-q} c_{11 u}^{q}-c_{21 l}^{1-q} c_{21 u}^{q}\right] \int_{0}^{t} \mathbb{E}\left|y_{1}(s ; q ; \eta)-y_{1}(s ; q ; \psi)\right| d s} \\
& \quad+\left[c_{22 l}^{1-q} c_{22 u}^{q}-c_{12 l}^{1-q} c_{12 u}^{q}\right] \int_{0}^{t} \mathbb{E}\left|y_{2}(s ; q ; \eta)-y_{2}(s ; q ; \psi)\right| d s \leqslant V(0)<\infty
\end{aligned}
$$

Thereby,

$$
\mathbb{E}\left|y_{i}(t ; q ; \eta)-y_{i}(t ; q ; \psi)\right| \in L^{1}[0, \infty), i=1,2
$$

According to model (1.3), one obtains

$$
\begin{aligned}
\mathbb{E}\left(y_{i}(t ; q)\right)= & y_{i}(0)+\int_{0}^{t}\left[r_{i l}^{1-q} r_{i u}^{(q)} \mathbb{E}\left(y_{i}(s ; q)\right)-c_{i i l}^{1-q} c_{i i u}^{q} \mathbb{E}\left(y_{i}^{2}(s ; q)\right)\right. \\
& \left.-c_{i j l}^{1-q} c_{i j u}^{q} \mathbb{E}\left(y_{i}(s ; q) y_{j}\left(s-\tau_{i l}^{1-q} \tau_{i u}^{q} ; q\right)\right)\right] d s, i, j=1,2, j \neq i .
\end{aligned}
$$

That is to say, $\mathbb{E}\left(y_{i}(t ; q)\right)$ is continuously differentiable. In addition, by virtue of (2.2),

$$
\frac{d \mathbb{E}\left(y_{i}(t ; q)\right)}{d t} \leqslant r_{i l}^{1-q} r_{i u}^{(q)} \mathbb{E}\left(y_{i}(t ; q)\right) \leqslant K_{1}, i=1,2,
$$

where $K_{1}$ is a positive constant. Therefore, $\mathbb{E}\left(y_{i}(t ; q)\right)$ is uniformly continuous. An application of Barbalat's conclusion [2] leads to the required assertion (3.2).

Part 2. In this part let us prove that there is a unique probability measure $v$ with support $R_{+}^{2}$ such that for any initial data $\eta \in \Gamma$, the transition probability $p(t, \eta, ; q)$ of $y(t ; q)$ converges weakly to $v$ as $t \rightarrow+\infty$.

Denote by $\mathcal{P}(t, \eta, A ; q)$ the probability of $y(t ; q ; \eta) \in A$. According to (2.2) and Chebyshev's inequality, one can observe that the family of $\{p(t, \eta, ; q)\}$ is tight. Let $\mathbb{P}(\Gamma ; q)$ be all the probability measures on $\Gamma$. For all $\mathrm{P}_{1}, \mathrm{P}_{2} \in \mathbb{P}$, define

$$
D_{H}\left(P_{1}, P_{2}\right)=\sup _{h \in H}\left|\int_{\mathbb{R}_{+}^{2}} h(x) P_{1}(d x)-\int_{\mathbb{R}_{+}^{2}} h(x) P_{2}(d x)\right|,
$$


where

$$
H=\{h: \Gamma \rightarrow \mathbb{R}|| h(x)-h(z)|\leqslant\|x-z\|,| h(\cdot) \mid \leqslant 1\} .
$$

For any $h \in H$ and $t, s>0$,

$$
\begin{aligned}
|\mathbb{E h}(y(t+s ; q ; \eta))-\mathbb{E h}(y(t ; q ; \eta))| & =\left|\mathbb{E}\left[\mathbb{E}\left(h(y(t+s ; q ; \eta)) \mid \mathcal{F}_{s}\right)\right]-\mathbb{E h}(y(t ; q ; \eta))\right| \\
& =\left|\int_{\mathbb{R}_{+}^{2}} \mathbb{E h}(y(t ; q ; \xi)) p(s, \eta, d \xi)-\mathbb{E h}(y(t ; q ; \eta))\right| \\
& \leqslant \int_{\mathbb{R}_{+}^{2}}|\mathbb{E h}(y(t ; q ; \xi))-\mathbb{E h}(y(t ; q ; \eta))| p(s, \eta, d \xi ; q) .
\end{aligned}
$$

It then follows from (3.2) that there is a $T$ such that for $t \geqslant T$,

$$
\sup _{h \in H}|\mathbb{E h}(y(t ; q ; \xi))-\mathbb{E h}(y(t ; q ; \eta))| \leqslant \epsilon .
$$

In other words,

$$
|\operatorname{Eh}(y(t+s ; q ; \eta))-\mathbb{E h}(y(t ; q ; \eta))| \leqslant \epsilon .
$$

An application of the arbitrariness of $h$ yields

$$
\sup _{h \in H}|\operatorname{Eh}(y(t+s ; q ; \eta))-\mathbb{E h}(y(t ; q ; \eta))| \leqslant \epsilon .
$$

Consequently,

$$
D_{H}(p(t+s, \eta, ; q), p(t, \eta, ; q)) \leqslant \epsilon, \quad \forall t \geqslant T, s>0 .
$$

Namely, $\{p(t, \lambda, ; q): t \geqslant 0\}$ is Cauchy in $\mathbb{P}$, where $\lambda=\lambda(\theta) \equiv(0.2,0.2)^{\top}, \theta \in[-\tau, 0]$. That is to say there is a unique $v(\cdot ; q) \in \mathbb{P}(\Gamma)$ such that

$$
\lim _{t \rightarrow+\infty} D_{H}(p(t, \lambda, \cdot ; q), v(\cdot ; q))=0
$$

By (3.2),

$$
\lim _{t \rightarrow+\infty} D_{H}(p(t, \eta, ; q), p(t, \lambda, ; q))=0
$$

Thereby

$$
\lim _{t \rightarrow+\infty} D_{H}(p(t, \eta, ; q), v(\cdot ; q)) \leqslant \lim _{t \rightarrow+\infty} D_{H}(p(t, \eta, \cdot ; q), p(t, \lambda, \cdot ; q))+\lim _{t \rightarrow+\infty} D_{H}(p(t, \lambda, \cdot ; q), v(\cdot ; q))=0 .
$$

According to (iii) in Theorem 2.5, then we obtain the desired assertion.

Part 3. In this part, let us prove $v$ is ergodic and (3.1) holds. According to the uniqueness of $v$ and Corollary 3.4.3 in [5], we can obtain that $v(\cdot)$ is strong mixing. Hence Theorem 3.2.6 in [5] means $v(\cdot)$ is ergodic. An application of (3.3.2) in [5] gives

$$
\lim _{t \rightarrow \infty} t^{-1} \int_{0}^{t} y_{i}(s ; q) d s=\int_{\mathbb{R}_{+}^{2}} \xi_{i} v\left(d \xi_{1}, d \xi_{2} ; q\right), i=1,2 .
$$

This together with (2.7) means (3.1).

(b). Note that $\Delta_{1}(q) \Delta_{2}(q)<0$, without loss of generality, we suppose that $\Delta_{1}(q)<0, \Delta_{2}(q)>0$. According to (ii) in Theorem 2.5, the species 1 goes to extinction. Hence model (1.3) does not have a stationary distribution. 


\section{Numerical simulations, discussions, and conclusions}

In this paper, we proposed and studied a two-species delay stochastic competition model with imprecise parameters. Our main results are Theorem 2.5 and Theorem 3.3. Theorem 2.5 establishes the threshold between persistence and extinction for each species. Theorem 3.3 gives the sharp sufficient criteria for the existence of a unique ergodic stationary distribution of the model. To the best of our knowledge, this paper is the first attempt to study stochastic delay population models with imprecise parameters.

Our results show that the imprecise parameters have close relationships with the persistence, extinction and the existence of stationary distribution of the model. To see these more clearly, let us consider the following example (in the following example and simulations, the values of parameters are chosen hypothetically). We choose $r_{1 l}=0.2, r_{1 u}=0.3, r_{2 l}=0.15, r_{2 u}=0.2, c_{11 l}=0.4, c_{11 u}=0.5, c_{12 l}=0.1$, $c_{12 u}=0.2, c_{21 l}=0.2, c_{21 u}=0.3, c_{22 l}=0.3, c_{22 u}=0.4, \sigma_{11 l}^{2}=0.1, \sigma_{11 u}^{2}=0.2, \sigma_{1 j l}=\sigma_{1 j u}=0, j=2, \ldots, n$, $\sigma_{22 l}^{2}=0.2, \sigma_{22 u}^{2}=0.1, \sigma_{2 k l}=\sigma_{2 k u}=0, k=1,3, \ldots, n, \tau_{1 l}=\tau_{2 l}=5, \tau_{1 u}=\tau_{2 u}=6$. Hence Assumption 3.1 holds. The only difference between the conditions of Figs. 1 and 2 is that the value of $q$ is different.

(A) In Fig. 1, we choose $q=0.8$, then $\Delta_{1}(0.8)=0.0503>0$ and $\Delta_{2}(0.8)=0.0054>0$. According to (a) in Theorem 3.3, both $y_{1}$ and $y_{2}$ are persistent and the model has a unique ergodic stationary distribution $v$ and:

$$
\begin{aligned}
& \int_{\mathbb{R}_{+}^{2}} \xi_{1} v\left(\mathrm{~d} \xi_{1}, \mathrm{~d} \xi_{2} ; 0.8\right)=\lim _{\mathrm{t} \rightarrow+\infty}\left\langle\mathrm{y}_{1}(\mathrm{t} ; 0.8)\right\rangle=\frac{\Delta_{1}(0.8)}{\Delta(0.8)}=0.3799, \\
& \int_{\mathbb{R}_{+}^{2}} \xi_{2} v\left(\mathrm{~d} \xi_{1}, \mathrm{~d} \xi_{2} ; 0.8\right)=\lim _{\mathrm{t} \rightarrow+\infty}\left\langle\mathrm{y}_{2}(\mathrm{t} ; 0.8)\right\rangle=\frac{\Delta_{2}(0.8)}{\Delta(0.8)}=0.0404 .
\end{aligned}
$$

Fig. 1 (a) is a sample path of the model and Fig. $1(\mathrm{~b})$ is the distribution of the solution at $t=8000$ (see e.g., [4]).

(B) In Fig. 2, we choose $q=0.5$, then $\Delta_{1}(0.5)>0$ and $\Delta_{2}(0.5)=-0.0037<0$. It then follows from (b) in Theorem 3.3 that $y_{2}$ goes to extinction and $y_{1}$ is persistent:

$$
\lim _{t \rightarrow+\infty}\left\langle y_{1}(t ; 0.5)\right\rangle=\frac{b_{1}(0.5)}{c_{11}^{0.5} c_{11 u}^{0.5}}=0.3873 .
$$

And hence the model does not have a stationary distribution.

Comparing Figs. 1 and 2 we can see that the imprecise parameters have close relationships with the persistence, extinction, and the existence of stationary distribution of the model. In fact, in the above example, if $\mathrm{q}>\ln 1.8$, then the model has a unique ergodic stationary distribution; if $\mathrm{q}<\frac{\ln 1.8}{\ln 1.5}$, then the model does not have a stationary distribution.

Some topics deserve further consideration. Firstly, it is interesting to study some more realistic but more complex systems, for example, reaction diffusion ([1]). Secondly, it is interesting to investigate foodchain models or cooperation models [11, 27]. Thirdly, in this paper we only consider the two-species model. It is of interest to investigate the n-species model. In fact, we also attempt to investigate this problem. However, we can not establish the critical values between persistence and extinction for each species at present stage. Finally, it is useful to study what happens if Assumption 2.3 is not satisfied. In this case, $\Delta_{1}(q)>0$ and $\Delta_{2}(q)>0$ will not hold simultaneously. Similar to the proof of Theorem 2.5 , we can show that

(i') if $\Delta_{1}(q)>0$ and $\Delta_{2}(q)<0$, then (i) in Theorem 2.5 holds;

(ii') if $\Delta_{1}(q)<0$ and $\Delta_{2}(q)>0$, then (ii) in Theorem 2.5 holds.

However, what happens if $\Delta_{1}(\mathrm{q})<0$ and $\Delta_{2}(\mathrm{q})<0$ are still unknown. We leave all of these for future work. 


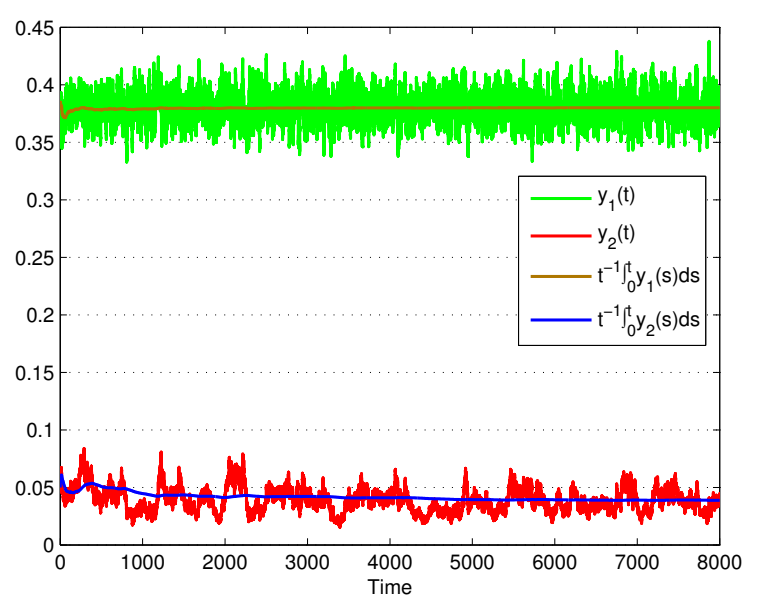

(a)

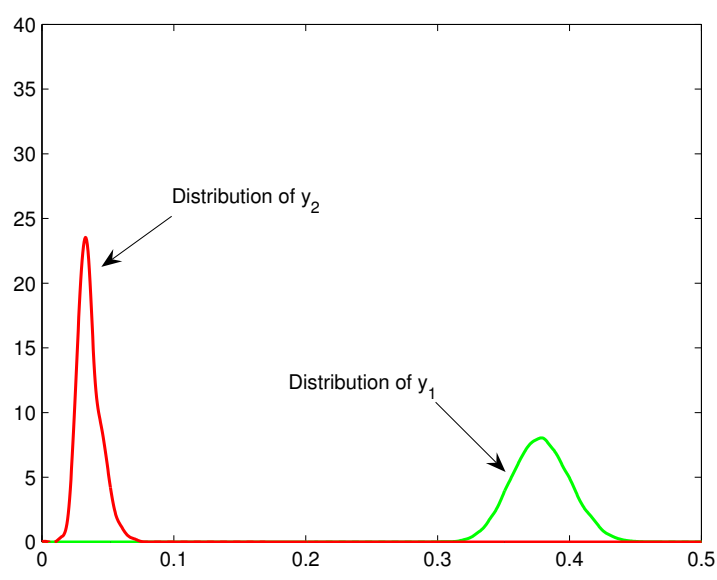

(b)

Figure 1: Model (1.3) with $q=0.8, r_{1 l}=0.2, r_{1 u}=0.3, r_{2 l}=0.15, r_{2 u}=0.2, c_{11 l}=0.4, c_{11 u}=0.5, c_{12 l}=0.1, c_{12 u}=0.2$, $c_{21 l}=0.2, c_{21 u}=0.3, c_{22 l}=0.3, c_{22 u}=0.4, \sigma_{11 l}^{2}=0.1, \sigma_{11 u}^{2}=0.2, \sigma_{1 j l}=\sigma_{1 j u}=0, j=2, \ldots, n, \sigma_{22 l}^{2}=0.2, \sigma_{22 u}^{2}=0.1$, $\sigma_{2 k l}=\sigma_{2 k u}=0, k=1,3, \ldots, n, \tau_{1 l}=\tau_{2 l}=5, \tau_{1 u}=\tau_{2 u}=6, \psi_{1}(\theta)=0.38+0.05 \sin \theta, \psi_{2}(\theta)=0.05+0.03 \sin \theta, \theta \in[-6,0] .(a)$ is a sample path; (b) is the distribution of the solution at $t=8000$.

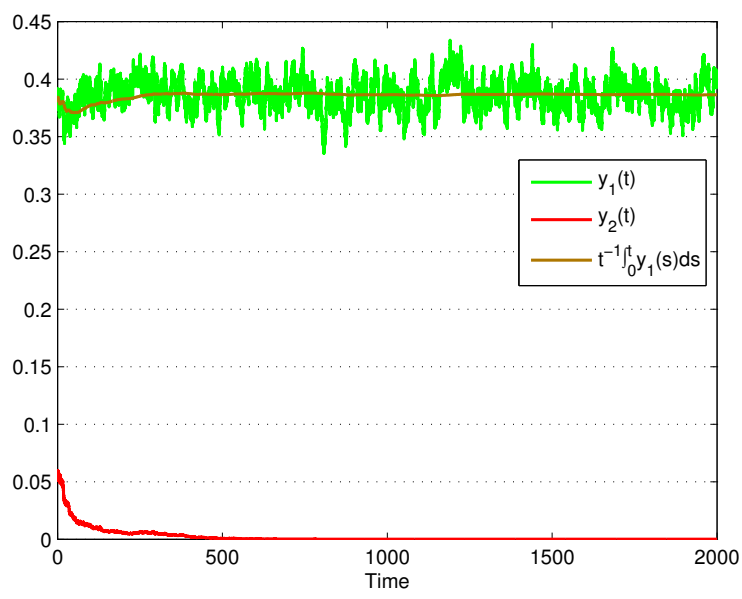

Figure 2: Model (1.3) with $q=0.5$, other parameters are the same with those in Fig. 1.

\section{Acknowledgment}

The authors thank Prof. Abdon Atangana and the anonymous referees for their careful reading and valuable comments, which greatly improved the presentation of the paper. The authors also thank the Natural Science Foundation of PR China (No. 11301207), Natural Science Foundation of Jiangsu Province (No. BK20170067), Jiangsu Province "333 High-Level Personnel Training Project" and "333 Project" Research Foundation in Jiangsu Province (No. BRA2017476), Jiangsu College Students Innovative Entrepreneurial Training Program (No. 201710323007Z).

\section{References}

[1] C.-Z. Bai, Multiplicity of solutions for a class of non-local elliptic operators systems, Bull. Korean Math. Soc., 54 (2017), 715-729. 4 
[2] I. Barbalat, Systémes d'équations différentielles d'oscillations non linéaires, (French) Rev. Math. Pures Appl., 4 (1959), 267-270. 3

[3] J. R. Beddington, R. M. May, Harvesting natural populations in a randomly fluctuating environment, Science, 197 (1977), 463-465. 1, 1.1

[4] Y.-L. Cai, Y. Kang, M. Banerjee, W.-M. Wang, A stochastic SIRS epidemic model with infectious force under intervention strategies, J. Differential Equations, 259 (2015), 7463-7502. 4

[5] G. Da Prato, J. Zabczyk, Ergodicity for infinite-dimensional systems, London Mathematical Society Lecture Note Series, Cambridge University Press, Cambridge, (1996). 3

[6] K. Gopalsamy, Stability and oscillations in delay differential equations of population dynamics, Mathematics and its Applications, Kluwer Academic Publishers Group, Dordrecht, (1992). 1

[7] N. Ikeda, S.Watanabe, A comparison theorem for solutions of stochastic differential equations and its applications, Osaka J. Math., 14 (1977), 619-633. 2

[8] D.-Q. Jiang, N.-Z. Shi, A note on nonautonomous logistic equation with random perturbation, J. Math. Anal. Appl., 303 (2005), 164-172. 2

[9] X.-Y. Li, X.-R. Mao, Population dynamical behavior of non-autonomous Lotka-Volterra competitive system with random perturbation, Discrete Contin. Dyn. Syst., 24 (2009), 523-545. 2

[10] M. Liu, C.-Z. Bai, Optimal harvesting of a stochastic logistic model with time delay, J. Nonlinear Sci., 25 (2015), $277-289$. 1

[11] M. Liu, C.-Z. Bai, Analysis of a stochastic tri-trophic food-chain model with harvesting, J. Math. Biol., 73 (2016), 597-625. 4

[12] M. Liu, C.-Z. Bai, Optimal harvesting of a stochastic delay competitive model, Discrete Contin. Dyn. Syst. Ser. B, 22 (2017), 1493-1508. 1

[13] M. Liu, C.-Z. Bai, Y. Jin, Population dynamical behavior of a two-predator one-prey stochastic model with time delay, Discrete Contin. Dyn. Syst., 37 (2017), 2513-2538. 1

[14] Q. Liu, Q.-M. Chen, Z.-H. Liu, Analysis on stochastic delay Lotka-Volterra systems driven by Lévy noise, Appl. Math. Comput., 235 (2014), 261-271. 1

[15] M. Liu, M. Fan, Stability in distribution of a three-species stochastic cascade predator-prey system with time delays, IMA J. Appl. Math., 82 (2017), 396-423. 1

[16] M. Liu, K. Wang, A note on a delay Lotka-Volterra competitive system with random perturbations, Appl. Math. Lett., 26 (2013), 589-594. 1

[17] M. Liu, K. Wang, Q. Wu, Survival analysis of stochastic competitive models in a polluted environment and stochastic competitive exclusion principle, Bull. Math. Biol., 73 (2011), 1969-2012. 2.1, 2

[18] X.-R. Mao, Stationary distribution of stochastic population systems, Systems Control Lett., 60 (2011), 398-405. 3

[19] R. M. C. May, Stability and complexity in model ecosystems, Princeton Univ. Press, Princeton, (2001). 1

[20] D. Pal, G. S. Mahapatra, Dynamic behavior of a predator-prey system of combined harvesting with interval-valued rate parameters, Nonlinear Dynam., 83 (2016), 2113-2123. 1

[21] D. Pal, G. S. Mahaptra, G. P. Samanta, Optimal harvesting of prey-predator system with interval biological parameters: a bioeconomic model, Math. Biosci., 241 (2013), 181-187. 1

[22] D. Pal, G. S. Mahaptra, G. P. Samanta, Stability and bionomic analysis of fuzzy parameter based prey-predator harvesting model using UFM, Nonlinear Dynam., 79 (2015), 1939-1955.

[23] D. Pal, G. S. Mahaptra, G. P. Samanta, Stability and bionomic analysis of fuzzy prey-predator harvesting model in presence of toxicity: a dynamic approach, Bull. Math. Biol., 78 (2016), 1493-1519.

[24] M. Peixoto, L. C. Barros, R. C. Bassanezi, Predator-prey fuzzy model, Ecol. Model., 214 (2008), $39-44$.

[25] S. Sharma, G. P. Samanta, Optimal harvesting of a two species competition model with imprecise biological parameters, Nonlinear Dynam., 77 (2014), 1101-1119. 1

[26] Y. Zhao, S.-L. Yuan, Q.-M. Zhang, Numerical solution of a fuzzy stochastic single-species age-structure model in a polluted environment, Appl. Math. Comput., 260 (2015), 385-396, 1

[27] Y. Zhu, M. Liu, Permanence and extinction in a stochastic service-resource mutualism model, Appl. Math. Lett., 69 (2017), 1-7. 4 